\title{
Augmented anti-angiogenesis activity of polysulfated heparin-endostatin and polyethylene glycol-endostatin in alkali burn-induced corneal ulcers in rabbits
}

\author{
ZHAO-NA LI ${ }^{1}$, ZHONG-FANG YUAN ${ }^{2}$, GUO-YING MU ${ }^{3}$, MING HU ${ }^{1}$, LI-JUN CAO ${ }^{1}$, \\ YA-LI ZHANG ${ }^{1}$ and MING-XU GE ${ }^{4}$
}

\begin{abstract}
${ }^{1}$ Department of Ophthalmology, Second People's Hospital of Jinan, Jinan, Shandong 250001; ${ }^{2}$ Department of Ophthalmology, Jinan Central Hospital, Shandong University, Jinan, Shandong 250013; Departments of ${ }^{3}$ Ophthalmology and ${ }^{4}$ Neurosurgery, Shandong Provincial Hospital, Shandong University, Jinan, Shandong 250021, P.R. China
\end{abstract}

Received August 2, 2014; Accepted June 5, 2015

DOI: $10.3892 /$ etm.2015.2602

\begin{abstract}
Endostatin (ES) is an endogenous angiogenesis inhibitor that has the ability to inhibit tumor growth and metastasis. However, its clinical application is limited by a number of disadvantages, such as poor stability, short half-life and the requirement of high doses to maintain its efficacy. The chemical modification on ES may offer a solution to these disadvantages. The aim of the present study was to evaluate the effects of ES, polysulfated heparin-endostatin (PSH-ES) and polyethylene glycol-endostatin(PEG-ES) on the endothelial cell proliferation and angiogenesis associated with corneal neovascularization $(\mathrm{CNV})$ and to determine their mechanisms of action. 3-(4,5-Dimethylthiazol-2-yl)-2,5-diphenyl-tetrazolium bromide (MTT) was used to study the effects of ES and its derivatives on endothelial cell proliferation in vitro, and rabbits were used to evaluate the effects of ES and its derivatives on $\mathrm{CNV}$ in vivo. In the evaluation of $\mathrm{CNV}$, the expression of vascular endothelial growth factor in the cornea was measured via immunohistochemistry and microvessels were counted. ES and its derivatives significantly inhibited endothelial cell proliferation in vitro $(\mathrm{P}<0.05)$ and suppressed $\mathrm{CNV}$ in vivo. Among the compounds examined, ES most effectively inhibited endothelial cell proliferation in vitro $(\mathrm{P}<0.05)$; however, PSH-ES and PEG-ES most effectively inhibited $\mathrm{CNV}$ in vivo $(\mathrm{P}<0.05)$. These results indicate that PSH-ES and PEG-ES are candidate anti-angiogenesis drugs.
\end{abstract}

Correspondence to: Dr Ming-Xu Ge, Department of Neurosurgery, Shandong Provincial Hospital, Shandong University, 324 Jingwuweiqi Road, Jinan, Shandong 250021, P.R. China

E-mail: gemingxu@126.com

Key words: endostatin, polysulfated heparin-endostatin, polyethylene glycol-endostatin, corneal neovascularization, vascular endothelial growth factor, endothelial cell proliferation

\section{Introduction}

Endostatin (ES) is an endogenous angiogenesis inhibitor that was initially identified by O'Reilly in 1997 (1). ES inhibits endothelial cell proliferation, migration and angiogenesis in the chorioallantoic membrane, and thus inhibits the growth and metastasis of tumors $(2,3)$. Furthermore, ES is able to reduce the resistance of tumors to chemotherapeutic agents with long-term repeated treatment. ES has previously attracted interest due to its capacity to treat retinal, choroidal and corneal neovascularization (CNV) in experimental settings (4). For example, the inhibition of CNV by intravenous injection of ES-expressing adenoviral vectors has been investigated, and microvessel development was observed to be inhibited (5). In September 2005, the State Food and Drug Administration of China approved the use of ES as an angiogenesis inhibitor for the treatment of patients with non-small-cell lung cancer (6). However, since ES is a protein, there are numerous obstacles preventing its effective clinical use as a drug, including the necessity for the administration of high doses $(7,8)$, short half-life, poor stability and high cost.

Recently, the chemical modification of protein drugs has become a topic of increased interest $(9,10)$. Modifications have been made with the aim of overcoming the inherent disadvantages of proteins and improving their effectiveness by prolonging half-life, lowering immunogenicity and increasing stability. In previous studies, ES has been chemically modified using polyethylene glycol (PEG) and polysulfated heparin (PSH), and the modified products have been found to exhibit enhanced heat stability, in addition to a high percentage of retained activity and limited alteration of the secondary structure $(11,12)$. PEG-ES and PSH-ES represent potential therapeutic agents for the treatment of cancer and other disorders that may have certain advantages in comparison with ES alone. However, to the best of our knowledge, the anti-angiogenesis effects of these modified proteins in the context of CNV have not yet been reported. Therefore, the present study investigated the efficacy of these compounds as inhibitors of endothelial cell proliferation and CNV, and compared their effects with those of ES. 


\section{Materials and methods}

Materials. PEG-6000 was purchased from Bio Basic, Inc. (Amherst, NY, USA). PSH was purchased from Yantai Dongcheng Biochemicals Co., Ltd. (Yantai, China). Pichia yeast containing the human ES gene was provided by the Medical School of Shandong University (Jinan, China). 3-(4,5-Dimethylthiazol-2-yl)-2,5-diphenyl-tetrazolium bromide (MTT) was purchased from Sigma-Aldrich (St. Louis, MO, USA). Human umbilical vein endothelial cells (HUVECs) were provided by the Institute of Pharmacology of Shandong University. Ready-to-use SP immunohistochemistry kits were purchased from Zhongshan Chuangyi Biochemical Engineering Co., Ltd. (Guangzhou, China). Basic fibroblast growth factor (bFGF) was purchased from Lanzhou Yisheng Biochemical Technology Co., Ltd. (Shanghai, China). New Zealand albino rabbits were obtained from the Laboratory Animal Department of Shandong University.

Preparation of ES. ES was prepared by a previously described method (11). In brief, ES was expressed in engineered Pichia yeast containing the human ES gene. The culture supernatant of the Pichia yeast was purified using carboxymethylcellulose-II exchange column chromatography (Whatman; GE Healthcare, Piscataway, NJ, USA) and Superdex 75 column chromatography (Pharmacia; GE Healthcare, Uppsala, Sweden). The resulting ES was assayed using SDS-PAGE and exhibited a single protein band at $20 \mathrm{kD}$.

Preparation of the PSH-ES conjugate. PSH was prepared and activated by periodate oxidation. In brief, heparin was dissolved in formamide at $60^{\circ} \mathrm{C}$ with constant stirring. A mixture of formamide and chlorosulfonic acid with a volume ratio of $2: 1$ was added to the heparin solution at $25^{\circ} \mathrm{C}$, and constantly stirred for $6 \mathrm{~h}$. The reaction was terminated by alcohol precipitation. Then, $0.5 \% \mathrm{NaHSO}_{3}$ was added to a $10 \%$ solution of the precipitate and the $\mathrm{pH}$ was adjusted to 9.0 with $\mathrm{Na}_{2} \mathrm{CO}_{3}$. The solution was then kept at $65^{\circ} \mathrm{C}$ for $3 \mathrm{~h}$, after which $1.0 \% \mathrm{NaCl}$ was added and the $\mathrm{pH}$ was adjusted to 6.5. The PSH $(0.3 \mathrm{~g})$ was dissolved in distilled water $(4.5 \mathrm{ml})$ and stirred while $12 \%$ sodium periodate solution $(0.5 \mathrm{ml})$ was added. The solution was adjusted from $\mathrm{pH} \sim 5.4$ to $\sim 5.0$ by the addition of hydrochloric acid $(0.1 \mathrm{~mol} / \mathrm{l})$. The solution was stirred in the dark for $20 \mathrm{~h}$. The activation reaction was then terminated by the dropwise addition of $5 \% \mathrm{NaHSO}_{3}$ solution. In the conjugation step, $90 \mathrm{mg}$ ES was dissolved in $1 \mathrm{ml}$ $0.3 \mathrm{M}$ sodium carbonate buffer ( $\mathrm{pH}$ 9.5), $6 \mathrm{ml}$ activated PSH (pH 9.0) was added and the solution was agitated slowly in the dark for $48 \mathrm{~h}$ at $4^{\circ} \mathrm{C}$. The reaction was terminated by adding glycine and the solution was subjected to Superdex 75 column chromatography (12). The eluent containing PSH-ES was subjected to filtration with a TX004 cellulose bag (Whatman) and then desalted with phosphate buffer (10 mM, pH 8.0) for $48 \mathrm{~h}$. Finally, the PSH-ES was subjected to concentration at $4^{\circ} \mathrm{C}$ and then was lyophilized.

Preparation of the PEG-ES conjugate. A mixture of $18 \mathrm{~g}$ PEG-6000, $2.2 \mathrm{~g}$ anhydrous $\mathrm{Na}_{2} \mathrm{CO}_{3}$ and $2.75 \mathrm{~g}$ cyanuric chloride was added to $75 \mathrm{ml}$ anhydrous benzene at room temperature and constantly stirred overnight. The solution was filtered and the product was precipitated with ethyl ether. The dissolution and precipitation step was repeated many times until no unreacted cyanuric chloride was detected by ultraviolet scanning. After vacuum drying, a white powder comprising activated PEG-6000 was obtained. ES and activated PEG-6000 were subsequently dissolved in $10 \mathrm{mM}$ sodium tetraborate buffer $(\mathrm{pH} 9.0)$ at a molar ratio of 1:40. The reaction was allowed to continue at $4^{\circ} \mathrm{C}$ under slow agitation for $24 \mathrm{~h}$, then glycine was added to terminate the reaction (13). The reaction solution was purified using carboxymethylcellulose-II exchange column chromatography and Superdex 75 column chromatography.

HUVEC proliferation assay. Culture was conducted as previously described (14). HUVECs were protected in Dulbecco's modified Eagle's medium (DMEM; Hyclone, Logan, UT, USA), which included 10\% heat-inactivated calf serum (Hyclone), $1 \%$ benzylpenicillin-streptomycin (Boehrvet, Germany) and $3 \mathrm{ng} / \mathrm{ml} \mathrm{bFGF}$. The inhibitory activity of ES, PSH-ES and PEG-ES on HUVEC proliferation in vitro was analyzed by MTT colorimetric analysis (15). Log-phase HUVECs were gathered and plated in a 96-well plate at $1.0 \times 10^{4}$ cells/well in a volume of $200 \mu \mathrm{l}$. These cells were incubated in a humidified atmosphere of $95 \%$ air $/ 5 \% \mathrm{CO}_{2}$ at $37^{\circ} \mathrm{C}$. Following the addition of ES, PSH-ES or PEG-ES $(5,10$ or $15 \mu \mathrm{g} / \mathrm{ml})$ to the wells, the cells were incubated at $37^{\circ} \mathrm{C}$ for $48 \mathrm{~h}$ in DMEM media with $10 \%$ calf serum. The supernatant was removed and HUVECs were washed twice with phosphate-buffered saline. Cells were resuspended in DMEM media with $10 \%$ calf serum and incubated with $20 \mu \mathrm{l}$ MTT solution $(5 \mathrm{mg} / \mathrm{ml})$ for $4 \mathrm{~h}$ at $37^{\circ} \mathrm{C}$. Subsequently, the supernatant was removed, $150 \mu \mathrm{l}$ dimethyl sulfoxide was added to each well and the plate was agitated for $10 \mathrm{~min}$. Absorbance was measured at $570 \mathrm{~nm}$ using a microplate reader (Model 680, Bio-Rad Laboratories, Hercules, CA, USA), and the inhibitory ratios (IR) were calculated as follows: IR $(\%)=(1-$ absorbance of experimental group/absorbance of blank control group) x 100 .

CNV assays. A study population of 32 New Zealand albino 6-month-old rabbits, 18 male and 14 female, was used in this study. Animals were treated in accordance with the Shandong University Animal Experimentation Ethic Committee (AEEC) guidelines and the study protocol was approved by the AEEC. All animal care, use and treatment were in strict accordance with the ARVO Statement for the use of Animals in Ophthalmic and Vision Research. Animals were anesthetized using a mixture of ketamine hydrochloride $(25 \mathrm{mg} / \mathrm{kg})$ and chlorpromazine $(25 \mathrm{mg} / \mathrm{kg})$ that was administered intramuscularly. Following the induction of sufficient anesthesia, as determined by corneal response, the central cornea of the right eye was burned by placing a $\mathrm{NaOH}$-soaked $(1 \mathrm{~mol} / \mathrm{l})$ circular piece of filter paper on the corneal surface for $60 \mathrm{sec}$. Following the removal of the filter paper, the ocular surface and the conjunctival sac were immediately rinsed with $20 \mathrm{ml}$ saline for $1 \mathrm{~min}$ and animals were allocated at random into four treatment groups ( $\mathrm{n}=8$ per group). Following ocular washing, $0.2 \mathrm{ml}$ PSH-ES (50 $\mu \mathrm{g} / \mathrm{ml})$, PEG-ES (50 $\mu \mathrm{g} / \mathrm{ml})$, ES $(50 \mu \mathrm{g} / \mathrm{ml})$ or physiological saline (as a control) were injected into the subconjunctival tissue. The animals received injections once every other day for 14 days, which was a total of 7 injections. Following each injection, topical antibiotic oint- 

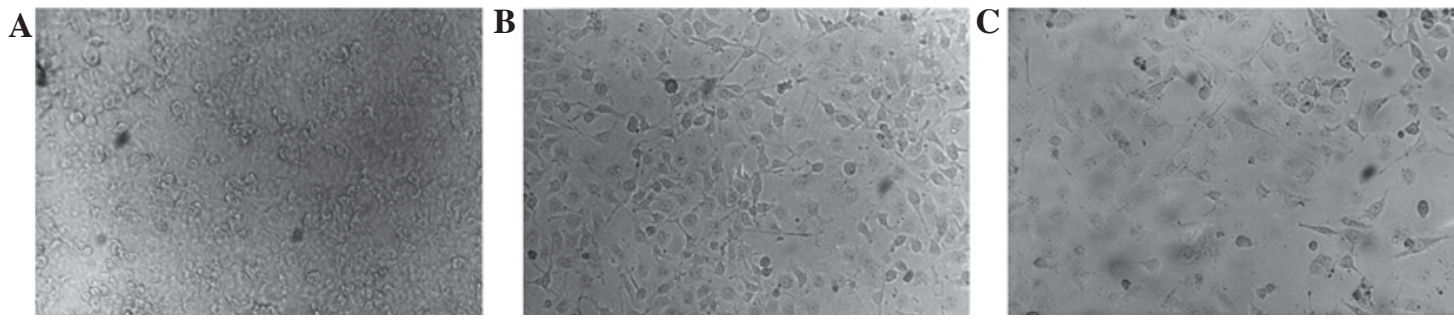

Figure 1. Inhibitory effects of ES, PSH-ES and PEG-ES on HUVEC proliferation. Purified ES, PSH-ES and PEG-ES (15 $\mu \mathrm{g} / \mathrm{ml})$ induced significant inhibitory effects on HUVEC proliferation. (A) ES, (B) PSH-ES and (C) PEG-ES groups (magnification, x400). ES, endostatin; PSH-ES, polysulfated heparin-endostatin; PEG-ES, polyethylene glycol-endostatin; HUVEC, human umbilical vein endothelial cell.

ment was administered to the burned eye to minimize the risk of infection.

The extent of CNV was quantified by the same observer using a slit lamp every day after the first injection. Furthermore, the ocular surface was examined for corneal ulceration and bulbar conjunctival hyperemia and edema. Rabbit eyes were photographed using a digital camera (Canon Inc., Tokyo, Japan) on days 4, 7, 10 and 13 and vessel growth from the corneoscleral limbus into the clear cornea was automatically quantified in terms of vessel area $\left(\mathrm{mm}^{2}\right)$ using Image-Pro Plus software (Media Cybernetics, Inc., Rockville, MD, USA) (16-18).

Rabbits were sacrificed viaan overdose of intravenous pentobarbital sodium 16 days after the alkali corneal burning. Eyes were enucleated and the cornea, including the adjacent $2 \mathrm{~mm}$ scleral tissue, was removed and immediately fixed in formalin. After $24 \mathrm{~h}$, tissue specimens were dehydrated, infiltrated and embedded in paraffin, and sectioned using a microtome. Serial olefin sections $(5 \mu \mathrm{m})$ of each eye were prepared and the expression of vascular endothelial growth factor (VEGF) was analyzed by immunohistochemical methods using goat antihuman VEGF (Santa Cruz Biotechnology, Santa Cruz, CA, USA). To visualize the vascular endothelial cells and determine the degree of induced angiogenesis, sections were stained with goat anti-rabbit CD34 antibody (Valeant Pharmaceuticals, Laval, Canada). The immunostaining process was performed according to the avidin-biotin complex (ABC) method. Thin paraffin sections $(5 \mu \mathrm{m})$ were mounted on silanized slides, dried overnight and deparaffinized with descending concentrations of ethanol and xylene. The slides were placed into citrate buffer ( $\mathrm{pH}$ 6.0) and boiled for $5 \mathrm{~min}$. After cooling for $30 \mathrm{~min}$, the specimens were incubated with $5 \%$ normal bovine serum for $30 \mathrm{~min}$, followed by incubation with the primary antibody in a humidified chamber for $2 \mathrm{~h}$ at room temperature. The primary antibodies used were goat anti-human VEGF antibody (1:400) and goat anti-rabbit CD34 antibody (1:400), plus $1 \%$ normal rabbit serum, respectively. For detection, the 3-step ABC method was used. The secondary biotinylated antibody was a mouse anti-goat immunoglobulin G (Abcam) antibody diluted 1:200. Finally, the slides were incubated with streptavidin and alkaline phosphatase and the samples were counterstained with Hoechst (1:1000; Sigma-Aldrich). Cell staining results were graded as 1 (yellow), 2 (brown) or 3 (sepia) based on the color of the cytoplasm. A Luzex-F image analyzer (Nireco Corporation, Tokyo, Japan) was used to obtain VEGF levels from the staining results, as indicated by grayscale levels. Three sections were selected for each eye,
Table I. Inhibitory effects of ES, PSH-ES, and PEG-ES on HUVEC proliferation.

\begin{tabular}{lccc}
\hline & \multicolumn{3}{c}{ Inhibitory rates (\%) } \\
\cline { 2 - 4 } Group & $5 \mu \mathrm{g} / \mathrm{ml}$ & $10 \mu \mathrm{g} / \mathrm{ml}$ & $15 \mu \mathrm{g} / \mathrm{ml}$ \\
\hline ES & 48.6 & 59.6 & 67.3 \\
PSH-ES & 42.2 & 45.4 & 56.8 \\
PEG-ES & 40.1 & 43.2 & 52.3 \\
\hline
\end{tabular}

ES, endostatin; PSH-ES, polysulfated heparin-endostatin; PEG-ES, polyethylene glycol-endostatin; HUVEC, human umbilical vein endothelial cell.

and the color of the cytoplasm was detected by the software at x400 magnification. Microvascular densities were determined using previously established methods (19). Three sections were selected for each eye, and new corneal vessels were counted in the 5 areas of highest vascular density at $x 400$ magnification. Microvascular density is expressed as the mean number of vessels per field of view area. Sections were also stained with hematoxylin and eosin for histological investigation.

Statistical analysis. Statistical analyses were performed using SPSS statistical software, version 15.0 (SPSS, Inc., Chicago, IL, USA). The statistical significance of the differences between the PSH-ES, PEG-ES, ES and control groups was determined using one-way analysis of variance and unpaired Student's t-tests. Data are presented as the mean \pm standard deviation and $\mathrm{P}<0.05$ was considered to indicate a statistically significant difference.

\section{Results}

Preparation of ES, PSH-ES and PEG-ES. The molecular weights of ES and its modified forms were determined using SDS-PAGE. ES, PSH-ES and PEG-ES presented with single bands at 20,35 and $38 \mathrm{kD}$, respectively, following purification. Final solutions of ES, PSH-ES and PEG-ES did not include visible fine particles or precipitate and remained clear at $4^{\circ} \mathrm{C}$ and after thawing from $-20^{\circ} \mathrm{C}$.

Effects of ES, PSH-ES and PEG-ES on HUVEC proliferation. In the cell proliferation assay, ES, PSH-ES and PEG-ES inhibited HUVEC proliferation in a concentration-dependent manner (Fig. 1 and Table I). The inhibitory rates of ES, 
Table II. Microvascular length and corneal neovascularization ( $\mathrm{n}=8$ eyes per group).

\begin{tabular}{|c|c|c|c|c|c|c|c|c|}
\hline \multirow[b]{2}{*}{ Group } & \multicolumn{2}{|c|}{ Day 4} & \multicolumn{2}{|c|}{ Day 7} & \multicolumn{2}{|c|}{ Day 10} & \multicolumn{2}{|c|}{ Day 13} \\
\hline & Length (mm) & Area $\left(\mathrm{mm}^{2}\right)$ & Length (mm) & Area $\left(\mathrm{mm}^{2}\right)$ & Length (mm) & Area $\left(\mathrm{mm}^{2}\right)$ & Length (mm) & Area $\left(\mathrm{mm}^{2}\right)$ \\
\hline PSH-ES & $0.42 \pm 0.12$ & $2.58 \pm 1.04$ & $1.38 \pm 0.16^{\mathrm{a}}$ & $4.39 \pm 1.27^{\mathrm{a}}$ & $2.15 \pm 0.96^{\mathrm{a}}$ & $5.08 \pm 1.97^{\mathrm{a}}$ & $2.32 \pm 1.46^{\mathrm{a}}$ & $10.18 \pm 3.26^{\mathrm{a}}$ \\
\hline PEG-ES & $0.51 \pm 0.16$ & $2.92 \pm 1.16$ & $1.56 \pm 0.19^{\mathrm{a}}$ & $5.02 \pm 1.18^{\mathrm{a}}$ & $2.34 \pm 0.93^{\mathrm{a}}$ & $6.88 \pm 2.02^{\mathrm{a}}$ & $2.97 \pm 1.54^{\mathrm{a}}$ & $12.37 \pm 3.92^{\mathrm{a}}$ \\
\hline ES & $0.88 \pm 0.21$ & $3.67 \pm 1.28$ & $1.73 \pm 0.28^{\mathrm{a}}$ & $5.82 \pm 1.24^{\mathrm{a}}$ & $2.68 \pm 1.07^{\mathrm{a}}$ & $8.59 \pm 2.08^{\mathrm{a}}$ & $3.49 \pm 1.68^{\mathrm{a}}$ & $16.89 \pm 4.15^{\mathrm{a}}$ \\
\hline Control & $1.35 \pm 0.86$ & $4.99 \pm 1.36$ & $2.38 \pm 0.56$ & $7.59 \pm 1.34$ & $3.92 \pm 1.29$ & $12.37 \pm 3.93$ & $5.85 \pm 1.28$ & $24.69 \pm 7.63$ \\
\hline
\end{tabular}

Data are presented as the mean \pm standard deviation. ${ }^{\mathrm{a}} \mathrm{P}<0.05$ vs. control group. PSH-ES, polysulfated heparin-endostatin; PEG-ES, polyethylene glycol-endostatin; ES, endostatin.

PSH-ES and PEG-ES were 48.6, 42.2 and 40.1\%, respectively, at a concentration of $5 \mu \mathrm{g} / \mathrm{ml} ; 59.6,45.4$ and $43.2 \%$, respectively, at $10 \mu \mathrm{g} / \mathrm{ml}$ and $67.3,56.8$ and $52.3 \%$, respectively, at $15 \mu \mathrm{g} / \mathrm{ml}$. ES produced more marked inhibition of HUVEC proliferation compared with PSH-ES and PEG-ES $(\mathrm{P}<0.05)$; however, no significant difference was observed between the effects produced by PSH-ES and PEG-ES $(\mathrm{P}<0.05)$.

Effects of ES, PSH-ES and PEG-ES in the CNV assay. Following alkali-induced corneal burning, the eyes of the rabbits were examined every day and photographic images captured under a surgical microscope. Corneal opacity and angiogenesis gradually increased in all groups; however, the ES, PSH-ES and PEG-ES groups exhibited significantly inhibited angiogenesis compared with the saline-treated control. The average CNV length in the ES, PSH-ES and PEG-ES groups was reduced compared with that in the saline group. Furthermore, the average CNV area in the ES, PSH-ES and PEG-ES groups was reduced compared with that in the saline group (Fig. 2 and Table II). The results indicate that the PSH-ES and PEG-ES derivatives inhibited angiogenesis in the CNV model more effectively than ES did.

It was hypothesized that the effects of PSH-ES and PEG-ES on CNV were mediated by the downregulation of VEGF, a known mediator of CNV and inflammation. Therefore, immunohistochemical analysis was used to measure corneal VEGF levels. In the physiological saline group, inflammatory cell infiltration occurred throughout the corneal hypothallus and the expression of VEGF in the inflammatory cytoplasm was grade 3 . In the ES group, inflammatory cell infiltration occurred in the anterior half of the corneal hypothallus and the expression of VEGF in the inflammatory cytoplasm was grade 2. In the PSH-ES and PEG-ES groups, inflammatory cell infiltration occurred in approximately one-third of the anterior of the corneal hypothallus and the expression of VEGF in the inflammatory cytoplasm was grade 1. VEGF expression was significantly inhibited by ES and modified ES, with PSH-ES and PEG-ES producing more marked effects compared with ES (P<0.05; Fig. 3 and Table II).

A limited number of new corneal vessels were detected in the eyes of rabbits treated with PSH-ES and PEG-ES. Additionally, the number of microvessels observed in the PSH-ES and PEG-ES groups on day 16 was significantly lower compared with that in the ES group. By contrast,
Table III. VEGF levels and the number of microvessels at day 16 ( $n=8$ eyes per group).

\begin{tabular}{lcc}
\hline Group & VEGF levels (grayscale) & Microvessels (n) \\
\hline PSH-ES & $101.32 \pm 17.46^{\mathrm{a}}$ & $1.04 \pm 0.82^{\mathrm{a}}$ \\
PEG-ES & $112.26 \pm 18.35^{\mathrm{a}}$ & $1.16 \pm 1.02^{\mathrm{a}}$ \\
ES & $123.56 \pm 20.13^{\mathrm{a}}$ & $3.59 \pm 1.75^{\mathrm{a}}$ \\
Saline & $153.15 \pm 24.54$ & $6.32 \pm 2.75$ \\
\hline
\end{tabular}

Data are presented as the mean \pm standard deviation. Microvessels were counted under $\mathrm{x} 400$ magnification. ${ }^{\mathrm{a}} \mathrm{P}<0.05$ vs. control (saline) group. VEGF, vascular endothelial growth factor; PSH-ES, polysulfated heparin-endostatin; PEG-ES, polyethylene glycol-endostatin; ES, endostatin.

numerous new corneal vessels were detected in the eyes of the control group, and were observed throughout the entire stroma $(\mathrm{P}<0.05$; Fig. 4 and Table III). The results of the CNV assay indicate that PSH-ES and PEG-ES exhibit more marked anti-angiogenesis activity compared with ES, and that PSH-ES was the most effective among the three proteins investigated.

\section{Discussion}

The anti-angiogenic activity of ES results in the inhibition of endothelial cell adhesion, migration, and proliferation, in addition to the induction of apoptosis (3). ES is among the most effective endogenous angiogenesis inhibitors. It has exhibited notable anti-angiogenesis and antitumor effects, for example in the therapy of non-small-cell lung cancer (20). In addition to its effects on tumors, ES has been observed to inhibit CNV (4). However, the clinical application of ES has been limited by the high dose requirement, short half-life and poor stability (21).

Chemical modification is a commonly used method of improving the properties of proteins. PEG is a modification agent used in numerous drugs approved by the US Food and Drug Administration, and is the most commonly used modifier of proteins. PSH is a polysaccharide with anti-angiogenesis and antitumor activity, which has also been reported to be a suitable modifier for improving the properties of ES (12). Based on the results of our previous research $(10,12)$, the present 

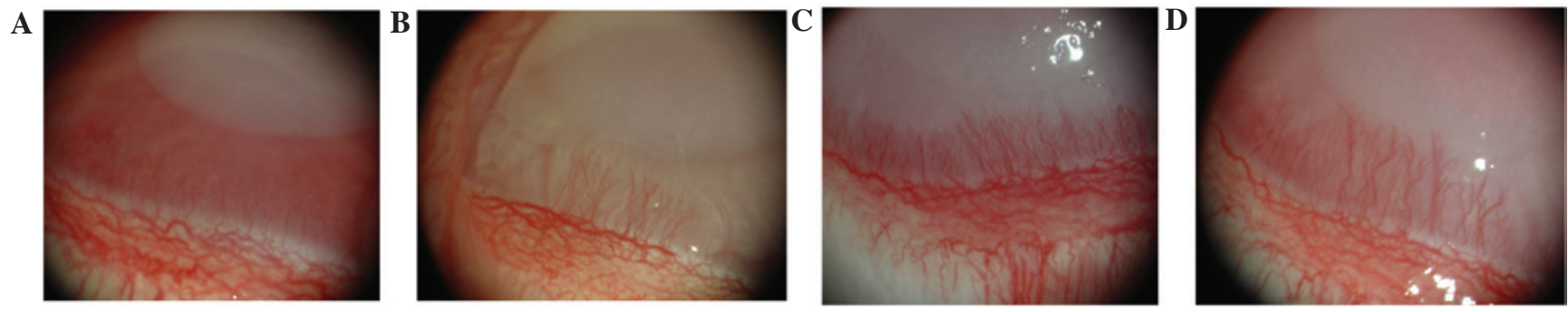

Figure 2. Effects of endostatin (ES), polysulfated heparin-endostatin (PSH-ES) and polyethylene glycol-endostatin (PEG-ES) on corneal neovascularization. (A) PSH-ES, (B) PEG-ES, and (C) ES (10 $\mu \mathrm{g} / \mathrm{eye})$ for 10 days, and (D) saline $(0.2 \mathrm{ml})$ for 10 days.
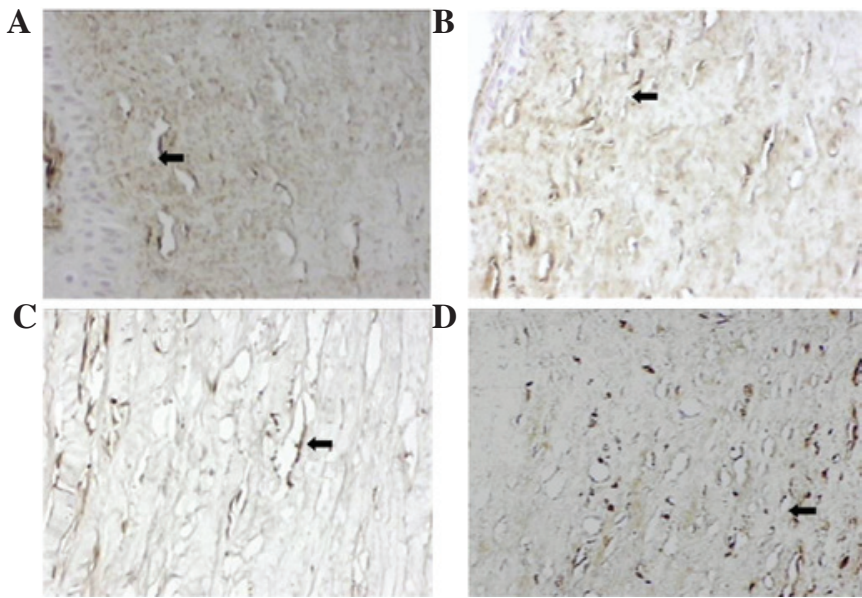

Figure 3. Vascular endothelial growth factor (VEGF) immunohistochemical assay. (A) Polysulfated heparin-endostatin, (B) polyethylene glycol-endostatin, (C) endostatin and (D) saline groups. The arrows indicate VEGF-positive staining in the luminal structures of endothelial cells (magnification, $\mathrm{x} 400)$.
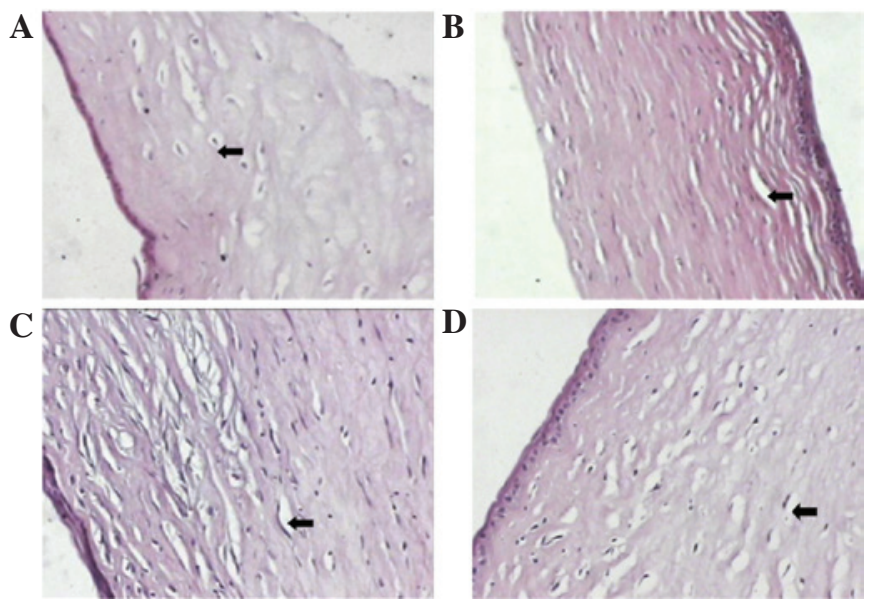

Figure 4. Histological examination of inflammatory cell infiltration and neovascularization. (A) Polysulfated heparin-endostatin, (B) polyethylene glycol-endostatin, (C) endostatin and (D) saline groups The arrows indicate new vessel growth (hematoxylin and eosin staining; magnification, x400) in the cornea.

study compared the anti-angiogenesis effects of ES and two ES derivatives.

The results of the HUVEC proliferation assay indicated that ES, PSH-ES and PEG-ES inhibited cell proliferation, with ES producing the most notable effect. Thus, purified
PSH-ES and PEG-ES exhibited reduced HUVEC inhibitory activity compared with ES, which may be due to alterations in its active domains following chemical modification. In addition, PSH-ES exhibited more pronounced antiproliferative activity compared with PEG-ES, which indicated that the PSH modification reduced activity to a lesser extent than the PEG modification.

The CNV assay indicated that PSH-ES produced the most marked inhibition of CNV, while PEG-ES produced more notable inhibitory effects compared with ES. Furthermore, VEGF expression was significantly inhibited by PSH-ES and PEG-ES. A previous study demonstrated that chemical modification is able to prolong protein half-life (22), and in our previous studies $(10,12)$, the stability of ES was improved by modification with PEG or PSH. It was hypothesized that the conjugation of ES with PSH or PEG may extend the biological half-life of ES (23) and thus prolong its effects. In a previous study, a chicken chorioallantoic membrane assay demonstrated that ES activity was prolonged by conjugation with PSH or PEG, with purified PSH-ES and PEG-ES exhibiting improved heat stability compared with ES at 25 and $37^{\circ} \mathrm{C}$ (24). These modification effects may underlie the notable in vivo anti-angiogenic properties of PSH-ES and PEG-ES, as compared with ES. Furthermore, the marked VEGF downregulation observed in the presence of PSH-ES and PEG-ES as compared with ES may have contributed to the higher efficacy of PSH-ES and PEG-ES in the inhibition of CNV.

The most commonly used protein modifier is currently PEG, which is not bioactive and produces no side-effects. The biological inactivity of PEG has resulted in its widespread use as a chemical modifier of proteins and peptides. However, in the present study the functional polysaccharide PSH was additionally used as an ES modifier, which increased the stability and bioactivity of ES, with no evident side-effects. This result may be due to the relatively large size of PSH-ES as compared with ES. The molecular weight of ES and the PSH modifier are 20 and $5.2 \mathrm{kD}$, respectively, as measured with gel permeation chromatography (12). SDS-PAGE has previously been used to demonstrate that the molecular weight of PSH-ES is $35 \mathrm{kD}$; therefore, it is presumed that, on average, one ES molecule conjugates with three PSH molecules. PSH-ES is structured with PSH on the compound exterior, where it protects ES and induces markedly improved stability compared with native ES. Finally, PSH possesses anti-angiogenic properties (24), and PSH and ES may function synergistically. Animal studies are currently underway to examine the immunogenicity and toxicity of endostatin if used independently and in combina- 
tion with chemotherapeutic agents. Collectively, previously published results (10) and the present study suggest that PSH-ES and PEG-ES may be clinically applicable interventions in the near future.

In the present study, ES and its derivatives PSH-ES and PEG-ES significantly inhibited HUVEC proliferation and CNV. Therefore, these conjugated ES compounds represent candidate anti-angiogenesis drugs that may lead to the expanded clinical application of ES derivatives for the treatment of angiogenesis-related diseases.

\section{Acknowledgements}

The authors thank Dr. Fengshan Wang of the Institute of Biochemical and Biotechnological Drugs at the Shandong University School of Pharmaceutical Science for technical support.

\section{References}

1. O'Reilly MS, Boehm T, Shing Y, et al: Endostatin: An endogenous inhibitor of angiogenesis and tumor growth. Cell 88: 277-285, 1997.

2. Addison CL, Nör JE, Zhao $\mathrm{H}$, et al: The response of VEGF-stimulated endothelial cells to angiostatic molecules is substrate-dependent. BMC Cell Biol 6: 38, 2005.

3. Dkhissi F, Lu H, Soria C, et al: Endostatin exhibits a direct antitumor effect in addition to its antiangiogenic activity in colon cancer cells. Hum Gene Ther 14: 997-1008, 2003.

4. Lai LJ, Xiao X and Wu JH: Inhibition of corneal neovascularization with endostatin delivered by adeno-associated viral (AAV) vector in a mouse corneal injury model. J Biomed Sci 14 313-322, 2007

5. Mori K, Ando A, Gehlbach P, et al: Inhibition of choroidal neovascularization by intravenous injection of adenoviral vectors expressing secretable endostatin. Am J Pathol 159: 313-320, 2001

6. Yang L, Wang JW, Sun Y, et al: Randomized phase II trial on escalated doses of rh-endostatin (YH-16) for advanced non-small cell lung cancer. Zhonghua Zhong Liu Za Zhi 28: 138-141, 2006 (In Chinese).

7. Zhuo W, Luo C, Wang X, et al: Endostatin inhibits tumour lymphangiogenesis and lymphatic metastasis via cell surface nucleolin on lymphangiogenic endothelial cells. J Pathol 222 249-260, 2010

8. Abdollahi A, Hlatky L and Huber PE: Endostatin: The logic of antiangiogenic therapy. Drug Resist Updat 8: 59-74, 2005.
9. Liu Z, Ren Y, Pan L and Xu HM: In vivo anti-tumor activity of polypeptide HM-3 modified by different polyethylene glycols (PEG). Int J Mol Sci 12: 2650-2663, 2011.

10. Tan H, Yang S, Liu C, et al: Enhanced anti-angiogenesis and anti-tumor activity of endostatin by chemical modification with polyethylene glycol and low molecular weight heparin. Biomed Pharmacother 66: 648-654, 2012.

11. Tan H, Yang S, Feng Y, et al: Characterization and secondary structure analysis of endostatin covalently modified by polyethylene glycol and low molecular weight heparin. J Biochem 144: 207-213, 2008

12. Ning TH, Chao CJ, Ying MG, et al: Preparation, characterization and anti-angiogenesis activity of endostatin covalently modified by polysulfated heparin. Pharmazie 67: 622-627, 2012.

13. Bullock J, Chowdhury S, Severdia A, et al: Comparison of results of various methods used to determine the extent of modification of methoxy polyethylene glycol 5000-modified bovine cupri-zinc superoxide dismutase. Anal Biochem 254: 254-262, 1997.

14. Han DW, Lee MH, Kim HH, et al: Epigallocatechin-3-gallate regulates cell growth, cell cycle and phosphorylated nuclear factor- $\kappa \mathrm{B}$ in human dermal fibroblasts. Acta Pharmacol Sin 32: 637-646, 2011.

15. Chen DQ, Wang X, Chen L, et al: Novel liver-specific cholic acid-cytarabine conjugates with potent antitumor activities: Synthesis and biological characterization. Acta Pharmacol Sin 32: 664-672, 2011.

16. Pan X, Wang Y, Zhang M, et al: Effects of endostatin-vascular endothelial growth inhibitor chimeric recombinant adenoviruses on antiangiogenesis. World J Gastroenterol 10: 1409-1414, 2004.

17. Igarashi T, Miyake K, Masuda I, et al: Adeno-associated vector (type 8)-mediated expression of soluble Flt-1 efficiently inhibits neovascularization in a murine choroidal neovascularization model. Hum Gene Ther 21: 631-637, 2010.

18. Yoshida M and Tabata Y: Tumor targeting of protein through poly (ethylene glycol) conjugation with metal coordination. J Nanosci Nanotechnol 10: 877-885, 2010.

19. Peng LH, Shen W, Yong W, et al: Effects of AMD3100 subconjunctival injection on alkali burn induced corneal neovascularization in mice. Int J Ophthalmol 4: 44-48, 2011.

20. Sun Y, Wang J, Liu Y, et al: Results of phase III trial of rh-endostatin (YH-16) in advanced nonsmall cell lung cancer (NSCLC) patients. J Clin Oncol 23 (Suppl): 7138, 2005.

21. Folkman J: Antiangiogenesis in cancer therapy - endostatin and its mechanisms of action. Exp Cell Res 312; 594-607, 2006.

22. Dosio F, Arpicco S, Brusa P, et al: Poly (ethylene glycol)-human serum albumin-paclitaxel conjugates: Preparation, characterization and pharmacokinetics. J Control Release 76: 107-117, 2001.

23. Zhu B, Xu HM, Zhao L, et al: Site-specific modification of anti-angiogenesis peptide HM-3 by polyethylene glycol molecular weight of $20 \mathrm{kDa}$. J Biochem 148: 341-347, 2010.

24. Norrby K: Low-molecular-weight heparins and angiogenesis. APMIS 114: 79-102, 2006. 